\title{
Erratum to "Prevalence of Sarcopenia among Nursing Home Older Residents in Cairo, Egypt" [Advances in Aging Research 3 (2014) 118-123]
}

\author{
Tomader Taha Abdel Rahman*, Hoda Mohammed Farid, Nehal Mahmoud Elkholy, \\ Ahmad Kamel Mortagy \\ Geriatrics Medicine Department, Faculty of Medicine, Ain Shams University, Cairo, Egypt \\ Email: tomelhagyn@hotmail.com
}

Received 15 March 2014; revised 16 April 2014; accepted 6 May 2014

Copyright (C) 2014 by authors and Scientific Research Publishing Inc.

This work is licensed under the Creative Commons Attribution International License (CC BY).

http://creativecommons.org/licenses/by/4.0/

\section{(c) (i) Open Access}

The original online version of this article (Rahman, T.T.A., et al. (2014) Prevalence of Sarcopenia among Nursing Home Older Residents in Cairo, Egypt. Advances in Aging Research, 3, $118-123$. http://dx.doi.org/10.4236/aar.2014.32019) was published in May.

\section{Abstract}

Sarcopenia is highly prevalent in community dwelling older adults in many countries; however, the prevalence of sarcopenia in nursing home older residents is not well characterized. The aim of this study is to assess the prevalence of sarcopenia in nursing home older residents in Cairo. Cross sectional study was performed among 150 nursing homes residents in Cairo. The European Working Group on Sarcopenia in Older People (EWGSOP) recommendation was used for diagnosing sarcopenia. The study showed that the prevalence of sarcopenia in nursing home older residents in Cairo was $17.3 \% ; 22.6 \%$ in elderly men and $13.6 \%$ in elderly women. Age, underweight and lack of physical activity were found to be associated with sarcopenia among studied participants. The study concluded that sarcopenia is an emerging health problem in nursing home older residents in Cairo.

\section{Results}

In this study, we used two handheld dynamometers. We discovered that one of them had a manufacturer defect and gave wrong results, so we retracted the patients' results done by this dynamometer. As well as we removed the data of the elderly who didn't complete all items of our assessment. So the studied participants were 150 participants and the results were as follow:

"Corresponding author. 
The mean age of studied participants was $69.8 \pm 6.9$ years. 58.7\% of participants were women. $46.0 \%$ were educated $\leq 12$ years and the rest were educated $>12$ years. As regard marital status $20.7 \%$ were married and $79.3 \%$ were singles. $22.7 \%$ were working physical work, $52.0 \%$ were working mental work and the rest had no previous occupation. Participants, BMI ranged between $17.5-25 \mathrm{~kg} / \mathrm{m}^{2} .56 .7 \%$ of participants do regular physical activity. $42.0 \%$ of participants have hypertension, $31.3 \%$ have diabetes mellitus, $18.7 \%$ have ischemic heart disease (IHD), 13.3\% have heart failure, $4.0 \%$ had previous history of stroke, $13.3 \%$ have osteoarthritis, 3.3\% have chronic liver disease (CLD), and 56.0\% have chronic obstructive pulmonary disease (COPD).

The prevalence of sarcopenia among nursing home older residents in Cairo was $17.3 \%$; $22.6 \%$ in elderly men and $13.6 \%$ in elderly women.

The study detected significant associations between sarcopenia and age $(\mathrm{p}=0.012)$ as well as between sarcopenia and BMI $(\mathrm{p}=0.025)$. Those with sarcopenia were less likely to be involved in regular physical activity $(\mathrm{p}=$ 0.019). There were no significant associations between sarcopenia and presence of co-morbidities $(\mathrm{p}>0.05)$ except for ischemic heart disease (IHD) which was significantly detected among residents with sarcopenia $(\mathrm{p}=$ 0.049) (Table 1).

\section{Discussion}

In the present study, we assessed the prevalence of sarcopenia among nursing home older residents in Cairo. Our findings showed that the prevalence of sarcopenia, using the EWGSOP recommendation, is $17.3 \%$; $22.6 \%$ in elderly men and $13.6 \%$ in elderly women.

Table 1. Characteristics of studied participants according to the presence of sarcopenia.

\begin{tabular}{|c|c|c|c|}
\hline & Non sarcopenic $(n=124)$ & Sarcopenic $(n=26)$ & $\mathrm{p}$ value \\
\hline Age (mean \pm SD) & $68.4 \pm 6.7$ & $74.3 \pm 8.9$ & 0.012 \\
\hline Men & $48(77.4 \%)$ & $14(22.6 \%)$ & 0.312 \\
\hline Education $\leq 12$ years & $54(78.3 \%)$ & $15(21.7 \%)$ & 0.430 \\
\hline Single & 99 (83.2\%) & $20(16.8 \%)$ & \multirow{2}{*}{0.821} \\
\hline Married & $25(80.7 \%)$ & $6(19.3 \%)$ & \\
\hline Physical work & 29 (85.3\%) & $5(14.7 \%)$ & \\
\hline Mental work & $66(84.6 \%)$ & $12(15.4 \%)$ & 0.612 \\
\hline No previous occupation & $29(76.3 \%)$ & $9(23.7 \%)$ & \\
\hline Body mass index, $\mathrm{kg} / \mathrm{m}^{2}$ (mean $\pm \mathrm{SD}$ ) & $20.8 \pm 3.7$ & $18.6 \pm 1.4$ & 0.025 \\
\hline Regular physical activity & $54(63.5 \%)$ & $31(36.5 \%)$ & 0.019 \\
\hline Fat free mass (mean $\pm \mathrm{SD}$ ) & $42.7 \pm 5.8$ & $36.6 \pm 4.3$ & 0.000 \\
\hline Fat free mass index (FFMI) (mean \pm SD) & $14.9 \pm 2.3$ & $11.3 \pm 2.4$ & 0.008 \\
\hline Hand grip (mean $\pm \mathrm{SD}$ ) & $19.2 \pm 8.0$ & $18.1 \pm 9.6$ & 0.357 \\
\hline Four meters walking test $(\mathrm{m} / \mathrm{s})($ mean $\pm \mathrm{SD})$ & $0.73 \pm 0.26$ & $0.62 \pm 0.31$ & 0.048 \\
\hline Hypertension & 49 (77.8\%) & $14(22.2 \%)$ & 0.521 \\
\hline Diabetes mellitus & $41(87.2 \%)$ & $6(12.8 \%)$ & 0.650 \\
\hline Ischemic heart disease & $11(39.3 \%)$ & $17(60.7 \%)$ & 0.049 \\
\hline Congestive heart failure & $11(55.0 \%)$ & $9(45.0 \%)$ & 0.136 \\
\hline Stroke & $6(100.0 \%)$ & $0(0.0 \%)$ & 0.385 \\
\hline Osteoarthritis & $18(90.0 \%)$ & $2(10.0 \%)$ & 0.421 \\
\hline Chronic liver disease & $4(80.0 \%)$ & $1(20.0 \%)$ & 0.943 \\
\hline Chronic obstructive pulmonary disease & $64(76.2 \%)$ & $20(23.8 \%)$ & 0.198 \\
\hline
\end{tabular}


In this study there is a significant association between age and sarcopenia $(p=0.012)$. This is consistent with the findings of previous studies [16]-[19] [22]. Aging is associated with significant changes in body composition, with a substantial reduction in fat free mass and an increase in visceral fat [12]. As well as, the age-dependent decrease in anabolic hormones, such as testosterone, estrogen, growth hormone, and insulin like growth factor-1 (IGF-1), may lead to loss of skeletal muscle mass [23] [24].

This study reported a significant association between BMI and sarcopenia $(p=0.025)$. This is consistent with the information reported by previous studies who documented the association between underweight and sarcopenia [4] [8] [25].

Previous studies [4] [26] [27] and the current study found a significant association between physical activity and sarcopenia $(p=0.019)$. Lack of physical activity has been shown to be a risk factor for muscle weakness that, in turn, results in loss of muscle mass and muscle strength [26] [27].

Previous studies [24] [28] as well as this study found that IHD was significantly associated with the presence of sarcopenia $(p=0.049)$. This may be due to the reported association between sedentary life style and the two diseases either IHD [29] or sarcopenia [26]. As well as, in IHD patients there is limitation in carrying out physical activities due to fear of ischemic chest pain or anticipation of pain [30]. This lack of physical activity will lead to sarcopenia.

\section{Conclusion}

The prevalence of sarcopenia using the EWGSOP recommendation was $22.6 \%$ in elderly men and $13.6 \%$ in elderly women. Age, underweight and lack of physical activity were found to be associated with sarcopenia in nursing home older residents in Cairo. 